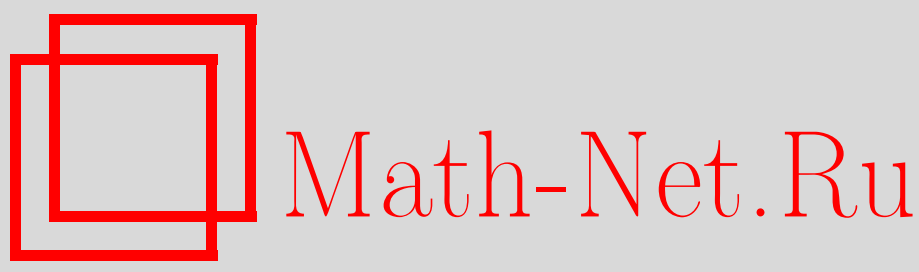

И. Ф. Красичков-Терновский, Аппроксимационная теорема для однородного уравнения векторной свертки, Матем. сб., 2004, том 195, номер 9, 37-56

DOI: https://doi.org/10.4213/sm844

Использование Общероссийского математического портала Math-Net.Ru подразумевает, что вы прочитали и согласны с пользовательским соглашением

http://www . mathnet.ru/rus/agreement

Параметры загрузки:

IP : 54.198 .187 .58

26 апреля 2023 г., $05: 15: 28$ 
УДК 517.59

\author{
И. Ф. Красичков-Терновский
}

\title{
Аппроксимационная теорема для однородного уравнения векторной свертки
}

Дается упрощенное доказательство известной аппроксимационной теоремы для однородного уравнения свертки. Используемый метод позволяет распространить эту теорему на более общий случай векторной свертки.

Библиограффия: 16 названий.

\section{Введение}

Пусть $\Omega$-выпуклая область в $\mathbb{C}^{n}, H(\Omega)$ - пространство функций, голоморфных в $\Omega$ с топологией равномерной сходимости на компактах в $\Omega, H^{*}(\Omega)$ - сильное сопряженное пространство к $H(\Omega)$. Любой линейный непрерывный функционал $S \in H^{*}(\Omega)$ допускает представление

$$
\langle S, f\rangle=\int f d \mu,
$$

где $\mu$ - комплексная конечно аддитивная мера ограниченной полной вариации с носителем, сосредоточенным на компакте $K \Subset \Omega$.

Пусть $\Omega \ominus K=\{h: h+K \subset \Omega\}$ - геометрическая разность множеств $\Omega$ и $K$. Это множество нам удобно обозначать через $O$. Нетрудно проверить, что $O$ - выпуклая окрестность нуля. Из представления (0.1) следует, что выражение $\langle S, f(z+h)\rangle$, где функционал действует по переменной $z$, представляет собой функцию, голоморфную в $O$. Эта функция называется сверткой функиионала $S$ u $\oint y н к и и и ~ f$ и обозначается $S * f$. Оператор свертки $f \rightarrow S * f$ линейно и непрерьвно отображает $H(\Omega)$ в $H(O)$. Рассмотрим однородное уравнение свертки

$$
S * f=0, \quad f \in H(\Omega) .
$$

Частным случаем уравнения (0.2) является однородное дифференциальное уравнение в частных производных с постоянными коэффициентами.

Экспоненциальным полиномом называется функция вида

$$
p(z) \exp \langle\lambda, z\rangle
$$

где $p(z)$ - многочлен. Экспоненциальные полиномы, удовлетворяющие уравнению (0.2), принято называть его әлементарными решениями. Возникает вопрос:

Работа вьполнена при поддержке Российского фонда фундаментальных исследований (грант № 02-01-00030). 
можно ли каждое решение уравнения (0.2) аппроксимировать в топологии $H(\Omega)$ линейными комбинациями элементарных решений? Положительный ответ называют аппроксимационной теоремой для уравнения свертки. В разных ситуациях аппроксимационная теорема была доказана следующими авторами: Л. Шварцем [1] $(\Omega=\mathbb{C})$, Л. Эренпрайсом [2], Б. Мальгранжем [3] $\left(\Omega=\mathbb{C}^{n}\right)$, И. Ф. Красичковым-Терновским [4] $(\Omega$ - выпуклая область в $\mathbb{C})$, В.В. Напалковым $[5](\Omega-$ трубчатая выпуклая область в $\left.\mathbb{C}^{n}\right)$, Р.С. Юлмухаметовым $[6](\Omega$ - выпуклая область в $\left.\mathbb{C}^{n}\right)$.

Среди этих утверждений наиболее сильное - это результат Р. С. Юлмухаметова [6]. Однако описанная им в краткой заметке [6] схема доказательства слишком фрагментарна, чтобы считаться удовлетворительной. Более развернутое описание той же схемы содержится в обзоре В.В. Напалкова и А. С. Кривошеева [7]. K сожалению, оно содержит неоднократные ссылки на мало доступные широкому читателю препринты [8], [9] Р. С. Юлмухаметова.

Таким образом, хотя аппроксимационная теорема хорошо известна специалистам, ее развернутое доказательство отсутствует в журнальной литературе. Цель настоящей статьи - восполнить этот пробел.

Известно, что аппроксимационная теорема сводится к так называемому “аналитическому" факту (см. [4]). В статьях [6], [7] аналитический факт интерпретируется в терминах специальной задачи весовой аппроксимации многочленами (см. [7; теорема 12.3]). Основная тяжесть рассуждений связана с этой задачей. В статьях [8], [9] весьма сложным путем задача весовой аппроксимации сводится к теореме Н. Сибони [10] о полиномиальной аппроксимации в интегро-весовых нормах.

В настоящей статье мы даем прямой и естественный метод вьвода аналитического факта из теоремы Сибони, минуя при этом упомянутую выше задачу весовой аппроксимации. Этот метод позволяет рассмотреть ситуацию более общую, чем описанная выше.

Вместо пространства $H(\Omega)$ мы рассматриваем топологическое произведение $\mathscr{H}=H\left(\Omega_{1}\right) \times \cdots \times H\left(\Omega_{\nu}\right) \quad \nu$ пространств такого типа. Линейный непрерывный функционал $S$ на этом пространстве представляется в виде вектора $S=$ $\left(S_{1}, \ldots, S_{q}\right)$, где $S_{i} \in H^{*}\left(\Omega_{i}\right)$; свертка функционала $S$ и элемента $f=\left(f_{1}, \ldots, f_{q}\right)$ представляется в виде суммы

$$
S * f=\sum_{i=1}^{q} S_{i} * f_{i} .
$$

Такую свертку, чтобы отличить ее от обычной, будем называть “векторной сверткой".

В работе доказывается аппроксимационная теорема для однородного уравнения векторной свертки.

\section{§1. Основные понятия}

1. Пусть $\Omega$ - область Рунге в $\mathbb{C}^{n}, H(\Omega)$ - пространство функций, голоморфных в $\Omega$, с топологией равномерной сходимости на компактах; $H(\Omega)$ есть пространство типа $\left(M^{*}\right)$ и как таковое является рефлексивным $[11] ; H^{*}(\Omega)$ - сопряженное к $H(\Omega)$ пространство, наделенное сильной топологией. Обозначим через $T$ преобразование Лапласа, которое каждому функционалу $S \in H^{*}(\Omega)$ ставит в соответствие его 
характеристическую функцию $\varphi(\lambda)=\langle S, \exp \langle\lambda, z\rangle\rangle ; P(\Omega)$ - образ $H^{*}(\Omega)$ относительно $T$. Поскольку $\Omega$ - область Рунге, отображение $T: H^{*}(\Omega) \rightarrow P(\Omega)$ является взаимно однозначньм; $P(\Omega)$ наделяется локально выпуклой топологией, индуцированной из $H^{*}(\Omega)$. Кроме того, $P(\Omega)$ выдерживает умножение на многочлены, причем операция умножения на фиксированный многочлен непрерывна в топологии $P(\Omega)$. Это позволяет рассматривать $P(\Omega)$ как топологический модуль над кольцом многочленов $\mathbb{C}[z]=\mathbb{C}\left[z_{1}, \ldots, z_{n}\right]$. Мы будем ссылаться на $P(\Omega)$ как на модуль, ассоциированный с областью $\Omega$.

В случае, когда область $\Omega$ выпуклая, топология $P(\Omega)$ может быть описана следуюшим образом. Пусть $D_{1} \Subset D_{2} \Subset \cdots-$ возрастаюшая последовательность выпуклых компактов, исчерпываюших $\Omega ; H_{D_{1}}<H_{D_{2}}<\cdots$ - последовательность опорных функций (в смысле комплексного анализа) этих компактов. Рассмотрим возрастающую последовательность банаховых пространств

$$
P_{1} \subset P_{2} \subset \cdots,
$$

где

$$
P_{n}=\left\{\varphi \in H\left(\mathbb{C}^{n}\right):\left\|\varphi_{n}\right\|=\sup _{\mathbb{C}^{n}} \frac{|\varphi(z)|}{\exp H_{D_{n}}(z)}<\infty\right\}
$$

Топология $P(\Omega)$ может быть описана как топология индуктивного предела этой последовательности. Так как вложения $P_{n} \subset P_{n+1}$ вполне непрерывны, то $P(\Omega)$ есть пространство типа $\left(L N^{*}\right)$ (см. [11]).

Из такого описания топологии в $P(\Omega)$ следует [11], что последовательность $\varphi_{n}$ сходится к $\varphi_{0}$ в топологии $P(\Omega)$ тогда и только тогда, когда

1) $\varphi_{n} \rightarrow \varphi_{0}$ равномерно на компактах,

2 ) сушествует компакт $K \Subset \Omega$ такой, что $\left|\varphi_{n}(z)-\varphi_{0}(z)\right| \leqslant$ const $\cdot \exp H_{K}(z)$.

2. Пусть $\Omega_{1}, \ldots, \Omega_{q}$ - система областей Рунге в $\mathbb{C}^{n}$;

$$
\mathscr{H}=H\left(\Omega_{1}\right) \times \cdots \times H\left(\Omega_{q}\right)
$$

- топологическое произведение; $\mathscr{H}$ является пространством типа $\left(M^{*}\right)$ и рефлексивным $[11] ; \mathscr{H}^{*}$ - сильное сопряженное к $\mathscr{H}$.

Линейный непрерывный функционал $S \in \mathscr{H}$ представляется в виде вектора $S=\left(S_{1}, \ldots, S_{q}\right)$ где $S_{j} \in H^{*}\left(\Omega_{j}\right), j=1, \ldots, q$. Действие функционала $S$ на элемент $f=\left(f_{1}, \ldots, f_{q}\right)$ осушествляется по правилу

$$
\langle S, f\rangle=\sum_{j=1}^{q}\left\langle S_{j}, f_{j}\right\rangle .
$$

Обозначим через $\mathscr{T}$ отображение, которое каждому функционалу

$$
S=\left(S_{1}, \ldots, S_{q}\right) \in \mathscr{H}^{*}
$$

ставит в соответствие его характеристический әлемент $\varphi=\left(\varphi_{1}, \ldots, \varphi_{q}\right)$, где $\varphi_{j}(\lambda)=\left\langle S_{j}, \exp \langle\lambda, z\rangle\right\rangle-$ характеристическая функция функционала $S_{j}, j=$ $1, \ldots, q$. 
Как и в случае $q=1$, отображение $\mathscr{T}$ будемназывать преобразованием Лаплаca. Обозначим через $\mathscr{P}$ образ $\mathscr{H}^{*}$ относительно $\mathscr{T}$. Так как $\Omega_{1}, \ldots, \Omega_{q}$ - области Рунге, то отображение $\mathscr{T}: \mathscr{H}^{*} \rightarrow \mathscr{P}$ является взаимно однозначньм. Наделим $\mathscr{P}$ топологией, индуцированной из $\mathscr{H}^{*}$. Из определения $\mathscr{P}$ следует, что $\mathscr{P}$ совпадает с топологическим произведением

$$
P\left(\Omega_{1}\right) \times \cdots \times P\left(\Omega_{q}\right) .
$$

Это влечет, что $\mathscr{P}$, как и каждое из пространств $P\left(\Omega_{1}\right), \ldots, P\left(\Omega_{q}\right)$, обладает структурой модуля над кольцом многочленов $\mathbb{C}[z]=\mathbb{C}\left(z_{1}, \ldots, z_{q}\right)$. Мы будем называть Я модулем, ассоииированным с системой областей $\Omega_{1}, \ldots, \Omega_{q}$.

3. Пусть $\Omega_{1}^{\prime}, \ldots, \Omega_{q}^{\prime}$ - еще одна система областей Рунге в $\mathbb{C}^{n}, \mathscr{H}^{\prime}=H\left(\Omega_{1}^{\prime}\right) \times$ $\cdots \times H\left(\Omega_{q}^{\prime}\right)$ и $u: \mathscr{H} \rightarrow \mathscr{H}^{\prime}-$ линейное непрерьвное отображение; $u^{*}: \mathscr{H}^{\prime *} \rightarrow \mathscr{H}^{*}-$ сопряженное отображение.

Обозначим через $W$ ядро операции $u, W^{0}=\left\{S \in \mathscr{H}^{*}:\langle S, W\rangle=0\right\}$ - подпространство в $\mathscr{H}^{*}$, "ортогональное" $W, V=u^{*}\left(\mathscr{H}^{\prime *}\right)$. Поскольку $u$ непрерывно, $V \subset \mathscr{H}^{*}$.

Обозначим через $\mathrm{Cl} V$ замыкание $V$ в $\mathscr{H}^{*}$.

ПРЕДЛОЖЕНИЕ 1.1. Справедливо соотношение

$$
\mathrm{Cl} V=W^{0} .
$$

ДоказАтельство. Обозначим через $\mathrm{cl} V$ слабое замыкание $V$ в $\mathscr{H}^{*}$. Известно, что $\mathrm{cl} V=W^{0}[12$; формула (8.6.4)]. Пространство $\mathscr{H}$ является рефлексивным. В этой ситуации слабое замыкание абсолютно выпуклого множества в $\mathscr{H}^{*}$ совпадает с его сильным замыканием. Множество $V$ как линейный образ абсолютно выпуклого множества $\mathscr{H}^{\prime *}$ является также абсолютно выпуклым. Поэтому cl $V=\mathrm{Cl} V$. Следовательно, $\mathrm{Cl} V=\mathrm{cl} V=W^{0}$.

Пусть $\mathscr{P}^{\prime}$ - модуль, ассоциированный с системой областей $\Omega_{1}^{\prime}, \ldots, \Omega_{q}^{\prime}$, $\mathscr{T}^{\prime}: \mathscr{H}^{\prime} \rightarrow \mathscr{P}^{\prime}-$ соответствующее преобразование Лапласа.

Преобразования Лапласа $\mathscr{T}, \mathscr{T}^{\prime}$ индуцируют операцию $u^{\circledast}: \mathscr{P}^{\prime} \rightarrow \mathscr{P}$, именно $u^{\circledast}=\mathscr{T} \circ u^{*} \circ \mathscr{T}^{\prime}-1$. Следуя [13], отображение $u^{\circledast}$ назовем дуальнымм по отношению к прямому отображению $u$. Обозначим $I=\mathscr{T}\left(W^{0}\right), J=u^{\circledast}\left(\mathscr{P}^{\prime}\right), \bar{J}-$ замыкание $J$ в топологии $\mathscr{H}$.

ПРЕДЛОЖЕНИЕ 1.2. Справедливо соотноиение

$$
\bar{J}=I .
$$

ДокАЗАТЕЛьство. Преобразование $\mathscr{T}$ есть топологический изоморфизм $\mathscr{H}^{*}$ на $\mathscr{P}$, причем $J=\mathscr{T}(V), I=\mathscr{T}\left(W^{0}\right)$. Поэтому соотношения (1.1) и (1.2) эквивалентны.

4. В пространстве $\mathscr{H}$ покомпонентно действуют операторы частного дифференцирования

$$
D_{1}=\frac{\partial}{\partial z_{1}}, \ldots, \quad D_{n}=\frac{\partial}{\partial z_{n}} .
$$


Элемент $f$ называется корневым әлементом системы операторов (1.3), отвечаюшим вектору собственных значений $\lambda=\left(\lambda_{1}, \ldots, \lambda_{n}\right)$, если при некотором целом $k$ вьполняются соотношения

$$
\left(D_{1}-\lambda_{1} E\right)^{k} f=0, \ldots, \quad\left(D_{n}-\lambda_{n} E\right)^{k} f=0,
$$

где $E$ - тождественный оператор.

В случае $q=1$, т.е. когда $f$ - скалярная функция, общее решение системы уравнений (1.4) представляет собой конечную линейную комбинацию функций вида

$$
z^{\alpha} \exp \langle C \lambda, z\rangle
$$

где $\alpha=\left(\alpha_{1}, \ldots, \alpha_{n}\right)$ - мультииндекс, $z^{\alpha}=z_{1}^{\alpha_{1}} \cdots z_{n}^{\alpha_{n}}$. В случае $q>1$ система (1.4) расщепляется на $q$ независимых систем

$$
\left(D_{1}-\lambda_{1} E\right)^{k} f_{j}=0, \ldots, \quad\left(D_{n}-\lambda_{n} E\right)^{k} f_{j}=0, \quad j=1, \ldots, q,
$$

где $f_{j} \in H\left(\Omega_{j}\right)$. Обшее решение такой системы можно представить в виде конечной линейной комбинации элементов из $\mathscr{H}$ вида

$$
z^{\alpha} \exp \langle\lambda, z\rangle e_{j},
$$

где $\alpha$-мультииндекс, $w \in \mathbb{C}^{n}, e_{j}$ - единичный $q$-вектор, у которого $j$-я компонента равна единице, а все остальные компоненты - нули.

5. Замкнутое подпространство $W \subset \mathscr{H}$ называется инвариантныцм, если оно инвариантно относительно системы операторов (1.3).

Пусть $W$ - инвариантное подпространство в $\mathscr{H}, W^{0}=\left\{S \in \mathscr{H}^{*}:\langle S, f\rangle=0\right.$ $\forall f \in W\}$ - подпространство в $\mathscr{H}^{*}$, “ортогональное" к $W$, и $I=\mathscr{T}\left(W^{0}\right) ; I$ обладает структурой замкнутого подмодуля $\mathbb{C}[z]$-модуля $\mathscr{P} ; I$ называется аннуляторнылм подмодулем подпространства $W$. Замкнутое инвариантное подпространство $W \subset \mathscr{H}$ допускает спектральныи синтез, если оно совпадает с замьканием в $\mathscr{H}$ множества конечных линейных комбинаций корневых элементов системы операторов (1.3), содержашихся в $W$.

6. Пусть $\lambda \in \mathbb{C}^{n} ; O(\lambda)$ - кольцо ростков функций $n$ переменных, аналитических в точке $\lambda ; \mathbb{O}(\lambda)$ - декартово произведение $q$ копий $O(\lambda)$. Будем рассматривать $\mathbb{O}(\lambda)$ как модуль над кольцом $O(\lambda)$. Всякий замкнутый подмодуль $I \subset \mathscr{P}$ порождает в $O(\lambda)$-модуле $\mathbb{O}(\lambda)$ локальный подмодуль $I(\lambda)$, состоящий из всевозможных комбинаций вида

$$
\sum c_{i} u^{(i)}
$$

где $u^{(i)} \in I, c_{i} \in O(\lambda)$.

Замкнутый подмодуль $I \subset \mathscr{P}$ называется обильныцм, если справедлива импликация

$$
f \in \mathscr{P}, \quad f \in I(\lambda) \quad \forall \lambda \in \mathbb{C}^{n} \Longrightarrow f \in I .
$$

ТЕОРЕМА ДВОЙСТВЕННОСТИ [14]. Замкнутое инвариантное подпространство $W \subset \mathscr{H}$ допускает спектральный синтез тогда и только тогда, когда его аннуляторный подмодуль $I \subset \mathscr{P}$ является обильныц.

Еше более общая теорема двойственности доказана А. Б. Шишкиным [15].

Эта теорема сводит задачу спектрального синтеза для инвариантного подпространства $W$ к проверке обильности его аннуляторного подмодуля $I=\mathscr{T}\left(W^{0}\right)$. 


\section{§2. Однородное уравнение векторной свертки}

1. Оператор векторной свертки. Фиксируем линейный непрерьвный функционал $S^{0}=\left(S_{1}^{0}, \ldots, S_{q}^{0}\right) \in \mathscr{H}^{*}$. Действие функционала $S^{0}$ на элемент $f=$ $\left(f_{1}, \ldots, f_{q}\right) \in \mathscr{H}$ осушествляется по правилу

$$
\left\langle S^{0}, f\right\rangle=\sum_{j=1}^{q}\left\langle S_{j}^{0}, f_{j}\right\rangle=\sum_{j=1}^{q} \int_{K_{j}} f_{j} d \mu_{j}
$$

где $\mu_{1}, \ldots, \mu_{q}$ - комплексные меры ограниченной вариации с носителями, сосредоточенными на компактах $K_{1} \subset \Omega_{1}, \ldots, K_{q} \subset \Omega_{q}$ соответственно.

Подберем области $G_{j}, j=1, \ldots, q$, такие, что $K_{j} \Subset G_{j} \Subset \Omega_{j}$. Существует открытая выпуклая окрестность нуля $O \subset \mathbb{C}^{n}$ такая, что $O+G_{j} \subset \Omega_{j}, j=$ $1, \ldots, q$. Для элемента $f=\left(f_{1}, \ldots, f_{q}\right) \in \mathscr{H}$ функции $f_{1}(z+h), \ldots, f_{q}(z+h)$ как функции переменных $z$ и $h$ будут аналитическими на множествах $G_{1} \times O, \ldots, G_{q} \times O$ соответственно. Поэтому при фиксированном $h \in O$ имеет смысл действие $S^{0}=$ $\left(S_{1}^{0}, \ldots, S_{q}^{0}\right)$ на вектор $f(z+h)=\left(f_{1}(z+h), \ldots, f_{q}(z+h)\right)$ :

$$
\left(S^{0} * f\right)(h):=\left\langle S^{0}, f(z+h)\right\rangle=\sum_{j=1}^{q} \int_{K_{j}} f_{j}(z+h) d \mu_{j}
$$

Это выражение представляет собой сумму сверток $S_{1} * f_{1}+\cdots+S_{\nu} * f_{\nu}$. Оно является функцией переменной $h$, голоморфной в $O$, и называется векторной сверткой функиионала $S^{0} \in H^{*}$ и әлемента $f=\left(f_{1}, \ldots, f_{q}\right) \in \mathscr{H}$.

Перечислим свойства оператора векторной свертки $u: f \rightarrow S * f$.

Свойство 1. Операция и есть линейное непрерьвное отображение $\mathscr{H}$ в $H(O)$.

Доказательство тривиально.

Свойство 2. Оператор свертки перестановочен с каждым из операторов $D_{1}, D_{2}, \ldots, D_{n}$.

Доказательство тривиально.

Пусть $P(O)$ - модуль, ассоциированный с областью $O$.

Свойство 3. Дуальная операция $u^{\circledast}: P(O) \rightarrow \mathscr{P}$ имеет вид $\varphi \rightarrow \varphi \varphi^{0}$, где $\varphi^{0}(\lambda)$ - характеристический элемент функиионала $S^{0}$.

ДоказАТеЛЬство. Сопряженная операция $u^{*}: H^{*}(O) \rightarrow \mathscr{H}^{*}$ переводит функционал $s \in H^{*}(O)$ в функционал $S \in \mathscr{H}^{*}$ по правилу

$$
\langle S, f\rangle=\langle s, u(f)\rangle=\left\langle s, S^{0} * f\right\rangle=\left\langle s, \sum_{j=1}^{q}\left(S_{j}^{0} * f_{j}\right)(h)\right\rangle=\sum_{j=1}^{q}\left\langle s, S_{j}^{0} * f_{j}\right\rangle,
$$


т.е. $S=\left(S_{1}, \ldots, S_{q}\right)$, где $\left\langle S_{j}, f\right\rangle=\left\langle s, S_{j}^{0} * f_{j}\right\rangle$. Отсюда следует, что дуальная операция $u^{\circledast}$ переводит характеристическую функцию $\varphi(\lambda)=\langle s, \exp \langle\lambda, z\rangle\rangle$ функционала $s$ в характеристический элемент $\psi=\left(\psi_{1}, \ldots, \psi_{q}\right)$ функционала $S=\left(S_{1}, \ldots, S_{q}\right)$ по правилу

$$
\begin{aligned}
\psi_{j}(\lambda) & =\left\langle S_{j}, \exp \langle\lambda, z\rangle\right\rangle=\left\langle s,\left\langle S_{j}^{0}, \exp \langle\lambda, z+h\rangle\right\rangle\right\rangle \\
& =\langle s, \exp \langle\lambda, h\rangle\rangle \cdot\left\langle S_{j}^{0}, \exp \langle\lambda, z\rangle\right\rangle=\varphi(\lambda) \varphi_{j}^{0}(\lambda), \quad j=1, \ldots, q .
\end{aligned}
$$

Таким образом, $\psi=\varphi\left(\varphi_{1}^{0}, \ldots, \varphi_{q}^{0}\right)=\varphi \varphi^{0}$. Свойство 3 установлено.

2. Однородное уравнение векторной свертки. Рассмотрим однородное уравнение свертки

$$
S * f=0, \quad f \in \mathscr{H} .
$$

ПрЕДЛОЖЕНИЕ 2.1. Множество $W$ решений $f \in \mathscr{H}$ уравнения (2.1) есть замкнутое инвариантное подпространство в $\mathscr{H}$.

ДоказАТЕЛЬСтво. То, что множество $W$ - замкнутое подпространство в $\mathscr{H}$, есть следствие линейности и непрерьвности оператора векторной свертки $u: \mathscr{H} \rightarrow$ $H(O)$ (свойство 1). Инвариантность $W$ относительно системы операторов $D_{i}$, $i=1, \ldots, n$, есть следствие перестановочности оператора $u$ с каждьм из операторов $D_{i}$ (свойство 2$)$.

Корневые элементы системы операторов $D_{1}, \ldots, D_{n}$, удовлетворяющие уравнению (2.1), называются әлементарными решениями этого уравнения.

Аппроксимационная теорема для однородного уравнения векторной свертки представляет собой

УТВеРЖДЕНИЕ. Каждое решение $f \in \mathscr{H}$ уравнения (2.1) аппроксимируется в топологии Н Н линейными комбинациями әлементарных решений; иными словами, инвариантное подпространство $W \subset \mathscr{H}$ решений уравнения (2.1) допускает спектральный синтез.

Мы докажем это утверждение в предположении, что система $\Omega=\left\{\Omega_{1}, \ldots, \Omega_{q}\right\}$ состоит из вьпуклых областей.

Замкнутый подмодуль $I \subset \mathscr{P}$ называется главным, порожденнылм әлементом $\varphi^{0} \in \mathscr{P}$, если он совпадает с замыканием в $\mathscr{P}$ множества всех элементов вида $p \varphi^{0}$, где $p \in \mathbb{C}[z]$.

ПрЕДЛОЖЕНИЕ 2.2. Аннуляторный подмодуль замкнутого инвариантного подпространства $W$ решений однородного уравнения векторной сверт$\kappa и(2.1)$ - главный подмодуль в Я્Р, порожденный характеристическим әлементом $\varphi^{0}=\left(\varphi_{1}^{0}, \ldots, \varphi_{n}^{0}\right)$ функиионала $S^{0}=\left(S_{1}^{0}, \ldots, S_{q}^{0}\right)$.

Лемма. Пусть $G, \Omega$ - области в $\mathbb{C}^{n} u G+O \subset \Omega$, әде $O$ - открытый шар с иентром в начале. Далее, пусть $\varphi^{(0)} \in P(G), l \in P(O)$ и существует последовательность многочленов $p_{n}, n=1,2, \ldots$, сходящаяся $x l$ в топологии $P(O)$. Тогда $p_{n} \varphi^{(0)}$ сходится $\kappa l \varphi^{(0)}$ в топологии $P(\Omega)$. 
ДокАЗАТЕльСтво. Включение $\varphi^{(0)} \in P(G)$ влечет представление $\varphi^{(0)}=$ $\langle S, \exp \langle\lambda, z\rangle\rangle$, где $S-$ линейный непрерывный функционал на $H(G)$. Этот функционал допускает представление

$$
\langle S, f\rangle=\int f d \mu
$$

где $\mu$ - комплексная конечно аддитивная мера ограниченной полной вариации, сосредоточенная на компакте $K \Subset G$. Таким образом, имеет место представление

$$
\varphi^{(0)}(\lambda)=\int \exp \langle\lambda, z\rangle d \mu
$$

Так как $K \Subset G$, то существует шар $O^{\prime}$ с центром в начале такой, что $K+O^{\prime} \subset G$. Компакт $K$ можно покрыть конечной системой открытых множеств $a_{1}+O^{\prime}, \ldots$, $a_{m}+O^{\prime}, a_{1}, \ldots, a_{m} \in K$. Меру $\mu$ можно представить в виде суммы $\mu=\mu_{1}+$ $\cdots+\mu_{m}$, где $\operatorname{supp} \mu_{i} \subset a_{i}+O^{\prime}, i=1, \ldots, m$. Из представления (2.2) следует, что

$$
\varphi^{(0)}(\lambda)=\varphi_{1}^{(0)}(\lambda)+\cdots+\varphi_{m}^{(0)}(\lambda)
$$

где $\varphi_{i}^{(0)}(\lambda)=\int f d \mu_{i}, i=1, \ldots, m$. Следовательно, в представлении (2.3) $\varphi_{i} \in$ $P\left(a_{i}+O^{\prime}\right)$. Далее, поскольку множества $a_{i}+O^{\prime}, O$ выпуклы,

$$
p_{n} \varphi_{i}^{(0)}(\lambda) \rightarrow l \varphi_{i}^{(0)}
$$

в топологии $P\left(a_{i}+O^{\prime}+O\right)$ (см. $\S 1$, п. 1$)$. Так как

$$
a_{i}+O^{\prime}+O \subset K+O^{\prime}+O \subset G+O \subset \Omega,
$$

то вложения $P\left(a_{i}+O^{\prime}+O\right) \subset P(\Omega)$ непрерывны. Поэтому сходимость $(2.4)$ будет иметь место и в топологии $P(\Omega)$. Это имеет место для любого $i=1, \ldots, m$. Поэтому последовательность

$$
p_{n} \varphi^{(0)}(\lambda)=p_{n} \varphi_{1}^{(0)}(\lambda)+\cdots+p_{n} \varphi_{m}^{(0)}(\lambda)
$$

сходится к $l \varphi_{1}^{(0)}(\lambda)+\cdots+l \varphi_{m}^{(0)}(\lambda)$ в топологии $P(\Omega)$. Лемма доказана.

ДОКАЗАТЕЛЬСТВО ПРЕДЛОЖЕНИЯ 2.2. При УсЛОВИИ $G_{i}+O \subset \Omega_{i}, i=1, \ldots, m$, оператор свертки будет линейно и непрерывно отображать $\mathscr{H}$ в $H(O)$. Подпространство $W$ решений $f \in \mathscr{H}(\Omega)$ уравнения (2.1) есть ядро оператора свертки

$$
u: f=\left(f_{1}, \ldots, f_{q}\right) \rightarrow S^{0} * f=\sum_{i=1}^{q} S_{i}^{0} * f_{i} .
$$

Пусть $J=u^{\circledast}(H(O))$ - образ $H(O)$ относительно дуальной операции $u^{\circledast}$. По свойству 3 (см. п. 1) $J$ состоит из элементов вида $\varphi \varphi^{(0)}$, где $\varphi^{(0)}=\left(\varphi_{1}^{(0)}, \ldots, \varphi_{q}^{(0)}\right)-$ 
характеристический элемент функционала $S^{0}=\left(S_{1}^{0}, \ldots, S_{q}^{0}\right)$, а $\varphi \in P(O)$. С другой стороны, по предложению 1.2 замыкание $J$ в топологии $\mathscr{P}$ совпадает с аннуляторным подмодулем $I=\mathscr{T}\left(W^{0}\right)$ инвариантного подпространства $W$. Из вышеизложенного следует: каждый элемент $\psi \in I$ можно аппроксимировать в топологии $\mathscr{P}$ элементами вида $\varphi \varphi^{(0)}, \varphi \in P(O)$. Далее, разлагая функцию $\varphi \in P(O)$ в ряд Тейлора и проделывая соответствующие оценки, убедимся, что существует последовательность многочленов $p_{n}, n=1,2, \ldots$, сходящаяся к $\varphi$ в топологии $P(O)$. В силу леммы последовательность $p_{n} \varphi_{i}^{(0)}$ сходится к $\varphi \varphi_{i}^{(0)}$ в топологии $P\left(\Omega_{i}\right)$. Поскольку такая сходимость имеет место для любого $i=1, \ldots, q$, заключаем, что $p_{n} \varphi^{(0)} \rightarrow \varphi \varphi^{(0)}$ в топологии $\mathscr{P}$. Из вышеизложенного следует, что каждьй элемент $\psi$ из $I$ аппроксимируется в топологии $\mathscr{P}$ элементами вида $p \varphi^{(0)}$, где $p$ - многочлен. Это означает, что $I$ есть главньй подмодуль в Я્Р. Предложение доказано.

3. Обильность главных подмодулей и аналитический факт. Рассмотрим главный подмодуль $I$ в $\mathscr{P}$, порождаемый элементом $\varphi^{(0)}$. Каждый элемент $u \in I$ аппроксимируется в топологии $\mathscr{P}$, а следовательно, и в более слабой топологии равномерной сходимости на компактах, элементами вида $p \varphi^{(0)}$, где $p$ - многочлен. Поэтому $u$ имеет вид $u=l \varphi^{(0)}$, где $\varphi^{(0)}$ - целая функция. Рассмотрим подмодуль $J \subset \mathscr{P}$, состоящий из всех элементов такого вида. Очевидно, $I \subset J$. Покажем, что $J$ - обильный подмодуль. По определению (см. $\S 1$, п. 6) локальный подмодуль $J(\lambda)$ состоит из конечных комбинаций

$$
\sum c_{i} u^{(i)}
$$

где $u_{i} \in J, c_{i} \in O(\lambda)$. Но $u^{(i)}=l^{(i)} \varphi^{(0)}$, где $l^{(i)}$ - целые функции. Поэтому сумма (2.5) есть $c \varphi^{(0)}$, где $c=\sum c_{i} l^{(i)} \in O(\lambda)$.

Итак, каждый локальный подмодуль $J(\lambda)$ состоит из элементов вида $c \varphi^{(i)}$, $c \in O(\lambda)$. Пусть теперь $f$ - элемент $\mathscr{P}$ такой, что $f \in J(\lambda) \forall \lambda \in \mathbb{C}^{n}$. Тогда в некоторой открытой окрестности $U_{\lambda}$ каждой точки $\lambda$ справедливо представление $f=c_{\lambda} \varphi^{(0)}$, где $c_{\lambda}$ голоморфна в $U_{\lambda}$. Если открытые множества $U_{\lambda}$ и $U_{\lambda^{\prime}}$ пересекаются, то в пересечении $U=U_{\lambda} \cap U_{\lambda^{\prime}}$ будет $c_{\lambda} \varphi^{(0)}=c_{\lambda^{\prime}} \varphi^{(0)}=f$, откуда следует, что $c_{\lambda}=c_{\lambda^{\prime}}$ в $U$. Отсюда стандартным образом следует, что семейство $\left\{c_{\lambda}\right\}$ склеивается в единую целую функцию $c$ и $f=c \varphi^{(0)}$. Таким образом, доказана импликация обильности:

$$
f \in \mathscr{P}, \quad f \in J(\lambda) \forall \lambda \in \mathbb{C}^{n} \Longrightarrow f \in J .
$$

Из вышеизложенного следует: для того чтобы доказать обильность $I$, достаточно доказать, что $I=J$. А для этого, ввиду включения $I \subset J$, достаточно установить, что каждый элемент $f \in J$ аппроксимируется в топологии $\mathscr{P}$ элементами вида $p \varphi^{(0)}$, где $p$ - многочлен. Итак, аппроксимационная теорема для однородного уравнения свертки будет доказана, если установить следующий

АНАЛИТИЧЕСКИЙ ФАКТ. Пусть әлементы $\varphi^{(0)}$ u $l \varphi^{(0)}$, әде $l-$ целая функция, принадлежсат Я્Р тогда существует последовательность многочленов $p_{n}, \quad n=1,2, \ldots$, такая, что $p_{n} \varphi^{(0)} \rightarrow l \varphi^{(0)}$ в топологии $\mathscr{P}$.

Отметим, что в ситуации $n=1, q=1$ аналитический факт был впервые сформулирован в [4].

Остальная часть статьи нацелена на доказательство аналитического факта. 


\section{§3. Аналитический факт}

В настоящем параграфе доказывается векторная версия аналитического факта. В случае $n=1$ такая версия была доказана ранее в $[16 ; \S 3]$.

ТЕОРЕМа 3.1. Пусть $l, \varphi_{1}, \ldots, \varphi_{q}-$ целье функиии, $\Omega_{1}, \ldots, \Omega_{q}-$ выпуклье области. Предположсим, что выполняется условие

$$
\varphi_{i} \in P\left(\Omega_{i}\right), \quad l \varphi_{i} \in P\left(\Omega_{i}\right), \quad i=1, \ldots, q
$$

Тогда существует последовательность многочленов $p_{\nu}, \quad \nu=1,2, \ldots$, сходящаяся $\kappa l$ равномерно на компактах и такая, что при каждом $i=1, \ldots, q$ $p_{\nu} \varphi_{i} \rightarrow l \varphi_{i}$ в топологии $P\left(\Omega_{i}\right)$.

Доказательство основано на теореме Н. Сибони [10; замечание к предложению 14].

1. Теорема Сибони. Обозначим через $\mathscr{L}$ класс плюрисубгармонических функций $\omega$ логарифмического роста, т.е. удовлетворяющих условию

$$
\omega(z) \leqslant a \ln ^{+}|z|+b
$$

где $a, b$ - положительные константы; $\mathscr{L}^{\prime}-$ класс верхних огибающих семейств функций из $\mathscr{L}$. Для произвольной функции $\omega \in \mathscr{L}^{\prime} \omega^{*}$ обозначает наименьшую полунепрерывную мажоранту функции $\omega$.

Следующее утверждение доказано Н. Сибони [10; замечание 15 к предложению 14].

Теорема СиБони. Пусть $F-$ целая функиия, а $\omega \in \mathscr{L}^{\prime}$. Если интеграл

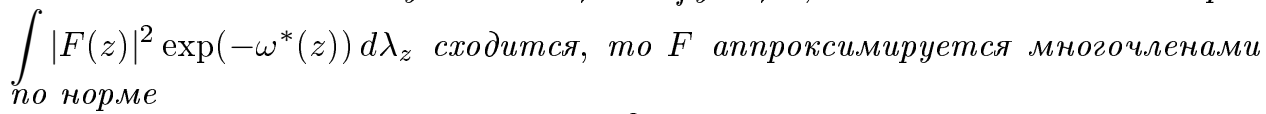

$$
\|f\|=\int \frac{|f(z)|^{2} \exp \left(-\omega^{*}\right)}{1+|z|^{2}} d \lambda_{z}
$$

где $\lambda_{z}-2 n$-мерная мера Лебега.

2. Мажорирование функциями класса $\mathscr{L}^{\prime}$. Следующее утверждение дает возможность воспользоваться теоремой Сибони.

ПРЕДЛОЖЕНИЕ 3.2. Пусть $F$ - челая функиия әкспоненщиального типа. Для любого $\varepsilon>0$ существует функиия $\omega$ класса $\mathscr{L}^{\prime}$ такая, что

$$
|F(z)| \leqslant \omega(z) \leqslant \ln ^{+}|F(z)|+\varepsilon|z|+\text { const. }
$$

Доказательство использует специальную оценку для частичных сумм ряда Тейлора.

Пусть $F$ - целая функция. Введем обозначения: $M_{F}(r)=\max _{|z| \leqslant r}|F(z)|-$ максимум модуля $F$,

$$
m_{F}(r)=\ln M_{F}(r), \quad \widetilde{m}_{F}(r)=\inf _{0<t \leqslant r}\left\{m_{F}(r+t)+n \ln \frac{r}{t}\right\} .
$$

Очевидно, $\tilde{m}_{F}(r) \leqslant m_{F}(r)$. 
АППРОКСИМАЦИОННАЯ ТЕОРЕМА ДЛЯ УРАВНЕНИЯ ВЕКТОРНОЙ СВЕРТКИ 47

Лемма. Пусть

$$
P_{\nu}(z)=\sum_{|\alpha| \leqslant \nu} a_{\alpha} z^{\alpha}
$$

-

$$
\ln \left|P_{\nu}(z)\right| \leqslant \widetilde{m}_{F}(r)+n \ln 2 .
$$

ДокАЗАТЕЛЬСТво. Пусть

$$
\widehat{M}_{F}\left(r_{1}, \ldots, r_{n}\right)=\max _{\left|z_{1}\right| \leqslant r_{1}, \ldots,\left|z_{n}\right| \leqslant r_{n}}|F(z)| .
$$

Легко видеть, что

$$
M_{F}(r)=\sup _{\sqrt{r_{1}^{2}+\cdots+r_{n}^{2}} \leqslant r} \widehat{M}_{F}\left(r_{1}, \ldots, r_{n}\right) .
$$

Используя неравенства Коши

$$
\left|a_{\alpha}\right| \leqslant \inf _{t_{1}, \ldots, t_{n}>0} \frac{\widehat{M}_{F}\left(t_{1}, \ldots, t_{n}\right)}{t_{1}^{\alpha_{1}} \cdots t_{n}^{\alpha_{n}}}
$$

имеем

$$
\left|P_{\nu}(z)\right| \leqslant \sum_{|\alpha| \leqslant \nu} \inf \frac{\widehat{M}_{F}\left(t_{1}, \ldots, t_{n}\right)}{t_{1}^{\alpha_{1}} \cdots t_{n}^{\alpha_{n}}} r_{1}^{\alpha_{1}} \cdots r_{n}^{\alpha_{n}} .
$$

Пусть $0<\varepsilon \leqslant 1$. Полагая $t_{1}=r_{1}(1+\varepsilon), \ldots, t_{n}=r_{n}(1+\varepsilon)$, получаем

$$
\begin{aligned}
\left|P_{n}(z)\right| & \leqslant \widehat{M}_{F}\left(r_{1}(1+\varepsilon), \ldots, r_{n}(1+\varepsilon)\right) \sum_{|\alpha|=0}^{\infty} \frac{1}{(1+\varepsilon)^{\alpha_{1}} \cdots(1+\varepsilon)^{\alpha_{n}}} \\
& =\widehat{M}_{F}\left(r_{1}(1+\varepsilon), \ldots, r_{n}(1+\varepsilon)\right)\left(\frac{1+\varepsilon}{\varepsilon}\right)^{n} \leqslant 2^{n} M_{F}(r(1+\varepsilon))\left(\frac{1}{\varepsilon}\right)^{n}
\end{aligned}
$$

Последнее неравенство справедливо в силу (3.3) и условия $0<\varepsilon \leqslant 1$. Итак,

$$
\left|P_{\nu}(z)\right| \leqslant 2^{n} \frac{M_{F}(r(1+\varepsilon))}{\varepsilon^{n}} .
$$

Это верно при любом $\varepsilon, 0<\varepsilon \leqslant 1$. Поэтому

$$
\begin{aligned}
\ln \left|P_{\nu}(z)\right| & \leqslant \inf _{0<\varepsilon \leqslant 1}\left(n \ln 2+m_{F}(r+\varepsilon r)+n \ln \frac{1}{\varepsilon}\right) \\
& =\inf _{0<t \leqslant r}\left(n \ln 2+m_{F}(r+t)+n \ln \frac{r}{t}\right)=n \ln 2+\widetilde{m}_{F}(r) .
\end{aligned}
$$

Лемма доказана.

Полагая $t=r$ в выражении $(3.2)$ для $\widetilde{m}_{F}(r)$, получаем неравенство

$$
\ln \left|P_{\nu}(z)\right| \leqslant m_{F}(2 r)+n \ln 2, \quad \nu=0,1, \ldots .
$$


ДОКАЗАТЕЛЬСТВО ПРЕДЛОЖЕНИЯ 3.2. По условию функция $F$ имеет экспоненциальный тип, т.е. существуют положительные константы $a, b$ такие, что

$$
m_{F}(r) \leqslant a r+b, \quad r>0 .
$$

Подбираем столь большое целое число $N$, что $1 / N<\varepsilon /(2 a)$. Представим $\ln |F|$ в виде

$\ln |F(z)|=\frac{1}{N} \ln |F(z)|+\cdots+\frac{1}{N} \ln |F(z)|=\frac{1}{N} \ln \left|F^{(1)}(z)\right|+\cdots+\frac{1}{N} \ln \left|F^{(N)}(z)\right|$, где $F^{(i)}(z) \equiv F(z), i=1, \ldots, N$.

В силу (3.4) и (3.5) частичные суммы $P_{\nu}(z)$ ряда Тейлора функции $F$ допускают оценку

$$
\ln \left|P_{\nu}(z)\right| \leqslant 2 a|z|+b+n \ln 2, \quad 0 \leqslant|z|<\infty .
$$

Фиксируем произвольное положительное число $R_{1}$. Пусть $P_{\nu_{1}}^{(1)}$ - частичная сумма ряда Тейлора функции $F^{(1)}$ такая, что $\left|F^{(1)}(z)-P_{\nu_{1}}^{(1)}(z)\right| \leqslant 1 / 2$ при $|z| \leqslant R_{1}$. Заметим, что $\left|P_{\nu_{1}}^{(1)}(z)\right| \leqslant\left|F^{(1)}(z)-P_{\nu_{1}}^{(1)}(z)\right|+\left|F^{(1)}(z)\right|$, откуда

$$
\ln \left|P_{\nu_{1}}^{(1)}(z)\right| \leqslant \ln \left(\frac{1}{2}+\left|F^{(1)}(z)\right|\right), \quad|z| \leqslant R_{1} .
$$

Если $\left|F^{(1)}(z)\right| \leqslant 1 / 2$, то $\ln \left|P_{\nu_{1}}^{(1)}(z)\right| \leqslant 0$; если $\left|F^{(1)}(z)\right|>1 / 2$, то

$$
\ln \left|P_{\nu_{1}}^{(1)}(z)\right| \leqslant \ln 2\left|F^{(1)}(z)\right|=\ln |F(z)|+\ln 2 .
$$

Таким образом, во всех случаях

$$
\ln \left|P_{\nu_{1}}^{(1)}(z)\right| \leqslant \ln ^{+}\left|F^{(1)}(z)\right|+1, \quad|z| \leqslant R_{1} .
$$

Положим

$$
\delta=\frac{\varepsilon}{2 a}-\frac{1}{N} .
$$

Очевидно, что $\delta>0$. Подбираем $R_{2}>R_{1}$ столь большим, что

$$
\ln \left|P_{\nu_{1}}^{(1)}(z)\right| \leqslant \delta(2 a|z|+b+n \ln 2), \quad|z|>R_{2} .
$$

Согласно (3.6) при $R_{1} \leqslant|z| \leqslant R_{2}$ будет выполняться оценка

$$
\ln \left|P_{\nu_{1}}^{(1)}(z)\right| \leqslant 2 a|z|+b+n \ln 2 .
$$

Итак, получаем следующие оценки:

$$
\begin{array}{lr}
\ln \left|P_{\nu_{1}}^{(1)}(z)\right| \leqslant \ln ^{+}\left|F^{(1)}(z)\right|+1, & |z| \leqslant R_{1} \\
\ln \left|P_{\nu_{1}}^{(1)}(z)\right| \leqslant 2 a|z|+b+n \ln 2, & R_{1} \leqslant|z| \leqslant R_{2} ; \\
\ln \left|P_{\nu_{1}}^{(1)}(z)\right| \leqslant \delta(2 a|z|+b+n \ln 2), & |z| \geqslant R_{2} .
\end{array}
$$


Теперь выберем частичную сумму $P_{\nu_{2}}^{(2)}$ ряда Тейлора $F^{(2)}$ так, чтобы

$$
\left|F_{1}^{(2)}(z)-P_{\nu_{2}}^{(2)}(z)\right| \leqslant \frac{1}{2}, \quad|z| \leqslant R_{2}
$$

Подбираем $R_{3}>R_{2}$ столь большим, чтобы

$$
\ln \left|P_{\nu_{2}}^{(2)}(z)\right| \leqslant \delta(2 a|z|+b+n \ln 2), \quad|z| \geqslant R_{3} .
$$

В силу (3.5) при $R_{2} \leqslant|z| \leqslant R_{3}$ выполняется неравенство

$$
\ln \left|P_{\nu_{2}}^{(2)}(z)\right| \leqslant 2 a|z|+b+n \ln 2 .
$$

Таким образом, $P_{\nu_{2}}^{(2)}$ допускает оценки

$$
\begin{array}{lr}
\ln \left|P_{\nu_{2}}^{(2)}(z)\right| \leqslant \ln ^{+}\left|F^{(2)}(z)\right|+1, & |z| \leqslant R_{2} ; \\
\ln \left|P_{\nu_{2}}^{(2)}(z)\right| \leqslant 2 a|z|+b+n \ln 2, & R_{2} \leqslant|z| \leqslant R_{3} ; \\
\ln \left|P_{\nu_{2}}^{(2)}(z)\right| \leqslant \delta(2 a|z|+b+n \ln 2), & |z| \geqslant R_{3} .
\end{array}
$$

Продолжая эту процедуру, построим систему чисел

$$
R_{1}<R_{2}<\cdots<R_{N+1}
$$

и систему многочленов

$$
P_{\nu_{1}}^{(1)}, \quad P_{\nu_{2}}^{(2)}, \ldots, P_{\nu_{N}}^{(N)}
$$

Обозначим

$$
P_{\nu_{1}, \ldots, \nu_{N}}(z)=P_{\nu_{1}}^{(1)}(z) \cdots P_{\nu_{N}}^{(N)}(z)
$$

При $|z| \leqslant R_{1}$ имеем

$$
\ln \left|P_{\nu_{1}}^{(1)}(z)\right| \leqslant \ln ^{+}\left|F^{(1)}(z)\right|+1, \quad \ldots, \quad \ln \left|P_{\nu_{N}}^{(N)}(z)\right| \leqslant \ln ^{+}\left|F^{(N)}(z)\right|+1,
$$

откуда следует

$$
\frac{1}{N} \ln \left|P_{\nu_{1}, \ldots, \nu_{N}}(z)\right|=\frac{1}{N}\left(\sum_{i=1}^{N} \ln ^{+}\left|F^{(i)}(z)\right|+1\right)=\ln ^{+}|F(z)|+1, \quad|z| \leqslant R_{1} .
$$

При $|z| \geqslant R_{N+1}$ имеем

$$
\ln \left|P_{\nu_{i}}^{(i)}(z)\right| \leqslant \delta(2 a|z|+b+n \ln 2)
$$

откуда

$$
\frac{1}{N} \ln \left|P_{\nu_{1}, \ldots, \nu_{N}}(z)\right| \leqslant \delta(2 a|z|+b+n \ln 2), \quad|z| \geqslant R_{N+1} .
$$


Оценим $\ln \left|P_{\nu_{1}, \ldots, \nu_{N}}(z)\right|$ при $R_{k} \leqslant|z| \leqslant R_{k+1}$. Имеем

$$
\begin{array}{rlrl}
\ln \left|P_{\nu_{i}}^{(i)}(z)\right| & \leqslant \delta(2 a|z|+b+n \ln 2), & & i<k ; \\
\ln \left|P_{\nu_{i}}^{(i)}(z)\right| \leqslant \frac{1}{N}\left(\ln ^{+}|F(z)|+1\right), & & i>k ; \\
\ln \left|P_{\nu_{k}}^{(k)}(z)\right| \leqslant(2 a|z|+b+\ln 2), & & i=k .
\end{array}
$$

Таким образом, при $R_{k} \leqslant|z| \leqslant R_{k+1}$ выполняется оценка

$$
\begin{aligned}
\frac{1}{N} & \ln \left|P_{\nu_{1}, \ldots, \nu_{N}}(z)\right|=\sum_{i=1}^{N} \frac{1}{N} \ln \left|P_{\nu_{i}}^{(i)}(z)\right| \\
& \leqslant \sum_{i<k} \frac{1}{N} \delta(2 a|z|+b+n \ln 2)+\frac{1}{N}(2 a|z|+b+n \ln 2)+\sum_{i>k} \frac{1}{N}\left(\ln ^{+}|F(z)|+1\right) \\
& =\frac{k-1}{N} \cdot \delta(2 a|z|+b+n \ln 2)+\frac{1}{N}(2 a|z|+b+n \ln 2)+\frac{N-k}{N}\left(\ln ^{+}|F(z)|+1\right) \\
& \leqslant\left(\ln ^{+}|F(z)|+1\right)+\left(\delta+\frac{1}{N}\right)(2 a|z|+b+n \ln 2) .
\end{aligned}
$$

Согласно (3.7) имеем $(\delta+1 / N) 2 a=\varepsilon$. Из оценок $(3.8),(3.9),(3.10)$ следует

$$
\frac{1}{N} \ln \left|P_{\nu_{1}, \ldots, \nu_{N}}(z)\right| \leqslant \ln ^{+}|F(z)|+\varepsilon|z|+b+n \ln 2 .
$$

Используя описанную выше процедуру, построим последовательность многочленов

$$
P_{\nu_{1}, \ldots, \nu_{N}}(z)=P_{\nu_{1}}^{(1)}(z) \times \cdots \times P_{\nu_{N}}^{(N)}(z),
$$

где $\nu_{1} \rightarrow \infty, \ldots, \nu_{N} \rightarrow \infty$. При этом

$$
P_{\nu_{1}}^{(1)} \rightarrow F(z), \quad \ldots, \quad P_{\nu_{N}}^{(N)}(z) \rightarrow F(z) .
$$

Отсюда

$$
\frac{1}{N} \sum \ln \left|P_{\nu_{i}}^{(i)}(z)\right| \rightarrow \ln |F(z)|
$$

Обозначим через $\omega$ верхнюю огибаюшую системы функций

$$
\frac{1}{N} \ln \left|P_{\nu_{1}}^{(1)} \times \cdots \times P_{\nu_{N}}^{(N)}\right|
$$

Из (3.12) следует, что $\omega(z) \geqslant \ln |F(z)|$, а из (3.11) следует, что $\omega(z) \leqslant \ln ^{+}|F(z)|+$ $\varepsilon|z|+b+n \ln 2$.

Предложение 3.2 доказано.

\section{3. Об отношении целых функций экспоненциального типа.}

ПРЕДЛОЖЕНИЕ 3.3. Пусть $\varphi, \Phi, l=\Phi / \varphi$ - целье функции. Если $\varphi, \Phi$ имеют әкспоненциальный тип, то и $l$ имеет әкспоненциальный тип.

Доказательство основано на лемме, которая имеет и самостоятельный интерес. 
ЛЕмма. Пусть $u, v, w=u-v-$ субгармонические функции на $\mathbb{R}^{m} ; \quad B-$ шар с чентром в точке $z$ радиуса меньше чем $|z|, E$ - наименьший шаровой слой с иентром в $z$, содержащий $B$.

Тогда

$$
w(z) \leqslant \frac{1}{|B|} \int_{B} u d \lambda+\frac{1}{|B|} \int_{E} v^{+} d \lambda-\frac{|E|}{|B|} v(0) .
$$

ДокАЗАТЕЛЬСтво. Если $v(0)=-\infty$, то неравенство (3.13) выполняется тривиальньм образом. Поэтому предположим, что $v(0)>-\infty$. В силу субгармоничности $w$ имеем

$$
w(z) \leqslant \frac{1}{|B|} \int_{B} w d \lambda=\frac{1}{|B|} \int_{B} u d \lambda-\frac{1}{|B|} \int_{B} v d \lambda .
$$

Оценим второй интеграл снизу. Обозначим через $v^{+}$и $v^{-}$положительную и отрицательную части функции $v$. Имеем

$$
\frac{1}{|B|} \int_{B} v d \lambda=\frac{1}{|B|} \int_{B}\left(v^{+}-v^{-}\right) d \lambda \geqslant-\frac{1}{|B|} \int_{B} v^{-} d \lambda \geqslant-\frac{1}{|B|} \int_{E} v^{-} d \lambda .
$$

Следовательно,

$$
w(z) \leqslant \frac{1}{|B|} \int_{B} u d \lambda+\frac{1}{|B|} \int_{E} v^{-} d \lambda=\frac{1}{|B|} \int_{B} u d \lambda+\frac{1}{|B|} \int_{E}\left(v^{+}-v\right) d \lambda .
$$

В силу субгармоничности $v$

$$
\frac{1}{|B|} \int_{E} v d \lambda=\frac{|E|}{|B|} \cdot \frac{1}{|E|} \int_{E} v d \lambda \geqslant \frac{|E|}{|B|} v(0)
$$

Из (3.14), (3.15) следует неравенство (3.13).

Лемма доказана.

ДОКАЗАТЕЛЬСТВО ПРЕДЛОЖЕНИЯ 3.3. БеЗ ограничения общности ПоЛожим $\varphi(0)=1$. Полагаем $u=\ln |\Phi|, v=\ln |\varphi|, w=\ln |l|$. Ясно, что $w=u-v$ и что $u, v, w$ - субгармонические функции, причем $v(0)=0$. Поскольку $\Phi, \varphi$ имеют экспоненциальный тип, сушествуют положительные константы $a, b$ такие, что

$$
u, v \leqslant a|z|+b .
$$

При $v(0)=0$ неравенство (3.13) принимает вид

$$
w(z) \leqslant \frac{1}{|B|} \int_{B} u d \lambda+\frac{1}{|B|} \int_{E} v^{+} d \lambda
$$

Фиксируем $\sigma, 0<\sigma<1$. В неравенстве (3.17) положим

$$
B=\{\xi:|\xi-z| \leqslant \sigma|z|\}, \quad E=\{\xi:|\xi-| z|| \leqslant \sigma|z|\} .
$$


Пусть $|B(1)|$ - объем единичного шара $B(1)=\{z:|z| \leqslant 1\}$. Вычислим объемы $B$ и $E$. Имеем

$$
\begin{gathered}
|B|=|B(1)|(\sigma|z|)^{2 n} \\
|E|=|B(1)|\left(|z|(1+\sigma)^{2 n}-|z|(1-\sigma)^{2 n}\right)=|B(1)|(|z| \sigma)^{2 n} C_{\sigma}
\end{gathered}
$$

где $C_{\sigma}=(1+1 / \sigma)^{2 n}-(1-1 / \sigma)^{2 n}$. Отсюда $|E| /|B|=C_{\sigma}$. Учитывая (3.16), имеем

$$
\begin{gathered}
\frac{1}{|B|} \int_{B} u d \lambda \leqslant \max _{B}|u| \leqslant a|z|(1+\sigma)+b ; \\
\frac{1}{|B|} \int_{E} v^{+} d \lambda \leqslant \frac{|E|}{|B|} \max _{E}\left|v^{+}\right| \leqslant C_{\sigma}(a|z|(1+\sigma)+b) .
\end{gathered}
$$

Отсюда согласно неравенству (3.17) получим неравенство

$$
\ln |l(z)|=w(z) \leqslant a^{\prime}|z|+b^{\prime},
$$

где $a^{\prime}=(1+\sigma) a\left(1+C_{\sigma}\right), b^{\prime}=b\left(1+C_{\sigma}\right)$.

Предложение 3.3 доказано.

Более сложным образом подобное утверждение было доказано Л. Эренпрай$\operatorname{com}[2]$.

4. Доказательство аналитического факта. Фиксируем число $\varkappa>n$. Из условия (3.1) следует, что сушествуют выпуклые компакты $K_{i}, K_{i} \Subset \Omega_{i}$, такие, что

$$
\ln \left|\varphi_{i}(z)\right|, \ln \left|\varphi_{i}(z) l(z)\right| \leqslant H_{K_{i}}(z)+\text { const }, \quad i=1, \ldots, q .
$$

Далее, подбираем компакты $K_{i}^{\prime}$ так, чтобы

$$
K_{i} \Subset K_{i}^{\prime} \Subset \Omega_{i}, \quad i=1, \ldots, q .
$$

Фиксируем $\varepsilon>0$ столь малое, что

$$
H_{K_{i}}(z)+\varepsilon|z|+\varkappa \ln ^{+}|z| \leqslant H_{K_{i}^{\prime}}(z)+\text { const. }
$$

Из неравенств (3.18), в частности, следует, что $\varphi_{i}, \varphi_{i} l$ имеют экспоненциальный тип. По предложению $3.3 l$ имеет также экспоненциальный тип. По предложению 3.2 существует функция $\omega \in \mathscr{L}^{\prime}$ такая, что

$$
\ln |l(z)| \leqslant \omega(z) \leqslant \ln ^{+}|l(z)|+\varepsilon|z|+\text { const. }
$$

Непосредственным вычислением можем убедиться, что форма Леви функции $\ln |z|$ неотрицательна, т.е. $\ln |z|$ - плюрисубгармоническая функция. Плюрисубгармонической будет и функция

$$
\ln ^{+}|z|=\max [1, \ln |z|] \text {. }
$$

Пусть $\omega_{0}(z)=\omega(z)+\varkappa \ln ^{+}|z|$. Легко видеть, что $\omega_{0} \in \mathscr{L}^{\prime}$ и

$$
\ln |l(z)|+\varkappa \ln ^{+}|z| \leqslant \omega_{0}(z) \leqslant \ln ^{+}|l(z)|+\varepsilon|z|+\varkappa \ln ^{+}|z|+\text { const. }
$$


Обозначим через $\omega_{0}^{*}$ наименьшую полунепрерывную сверху мажоранту $\omega_{0}$. Поскольку функция $\ln ^{+}|l(z)|+\varepsilon|z|+\varkappa \ln ^{+}|z|$ полунепрерывна сверху и мажорирует $\omega_{0}$, та же функция будет мажорировать и $\omega_{0}^{*}$. Таким образом, неравенства $(3.20)$ влекут неравенства

$$
\ln |l(z)|+\varkappa \ln ^{+}|z| \leqslant \omega_{0}^{*}(z) \leqslant \ln ^{+}|l(z)|+\varepsilon|z|+\varkappa \ln ^{+}|z|+\text { const. }
$$

Рассмотрим интеграл

$$
I=\int|l(z)|^{2} \exp \left(-2 \omega_{0}^{*}(z)\right) d \lambda_{z}
$$

Используя левое неравенство (3.21) и обозначая через $|S(1)|$ площадь $(2 n-1)$-мерной сферы, имеем

$$
\begin{aligned}
I & \leqslant \int|l(z)|^{2} \exp \left(-2\left(\ln |l(z)|+\varkappa \ln ^{+}|z|\right)\right) d \lambda_{z}=\int \exp \left(-2 \varkappa \ln ^{+}|z|\right) d \lambda_{z} \\
& =\text { const }+\int_{|z| \geqslant 1}|z|^{-2 \varkappa} d \lambda_{z}=\mathrm{const}+|S(1)| \int_{\rho \geqslant 1} \frac{1}{\rho^{2 \varkappa-2 n+1}} d \rho .
\end{aligned}
$$

По условию $2 \varkappa-2 n+1>1$. Поэтому интеграл (3.22) сходится.

Имеем $2 \omega^{*}=(2 \omega)^{*} \in \mathscr{L}^{\prime}$. Поэтому по теореме Сибони (см. $\S 3$, п. 1$)$ существует последовательность многочленов $P_{\nu}, \nu=1,2, \ldots$, такая, что

$$
\int \frac{\left|P_{\nu}(\xi)-l(\xi)\right|^{2}}{1+|\xi|^{2}} \exp \left(-2 \omega_{0}^{*}(\xi)\right) d \lambda_{\xi} \rightarrow 0
$$

при $\nu \rightarrow \infty$. Отсюда в силу правого неравенства (3.21) следует

$$
I_{\nu}=\int \frac{\left|P_{\nu}(\xi)-l(\xi)\right|^{2}}{\left(1+|\xi|^{2}\right)} \exp \left(-2\left(\ln ^{+}|l(\xi)|+\varepsilon|\xi|+\varkappa \ln ^{+}|\xi|\right)\right) d \lambda_{\xi} \rightarrow 0
$$

при $\nu \rightarrow \infty$.

Пусть $\varphi_{i}-$ одна из функций, участвующих в условии (3.1). Положим $\omega_{i}(\xi)=$ $\ln ^{+}|l(\xi)|+\ln \left|\varphi_{i}(\xi)\right|+\varepsilon|\xi|+\varkappa \ln ^{+}|\xi|$ и перепишем интеграл $I_{\nu}$ в виде

$$
I_{\nu}=\int \frac{\left|P_{\nu}(\xi)-l(\xi)\right|^{2}\left|\varphi_{i}(\xi)\right|^{2}}{1+|\xi|^{2}} \exp \left(-2 \omega_{i}(\xi)\right) d \lambda_{\xi}
$$

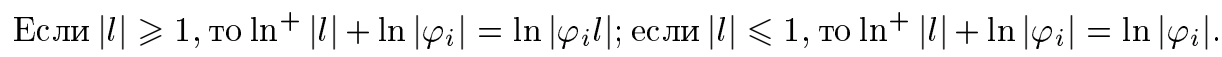
Таким образом, во всех случаях

$$
\ln ^{+}|l|+\ln \left|\varphi_{i}\right| \leqslant \max \left[\ln \left|\varphi_{i} l\right|, \ln |l|\right] .
$$

Отсюда в силу (3.18) и (3.19) имеем

$$
\begin{aligned}
\omega_{i}(\xi) & =\ln ^{+}|l(\xi)|+\ln \left|\varphi_{i}(\xi)\right|+\varepsilon|\xi|+\varkappa \ln ^{+}|\xi| \\
& \leqslant H_{K_{i}}(\xi)+\varepsilon|\xi|+\varkappa \ln ^{+}|\xi| \leqslant H_{K_{i}^{\prime}}(\xi)+\text { const. }
\end{aligned}
$$


Полагаем

$$
I_{\nu, i}=\int \frac{\left|P_{\nu}(\xi) \varphi_{i}(\xi)-l(\xi) \varphi_{i}(\xi)\right|^{2}}{1+|\xi|^{2}} \exp \left(-2 H_{K_{i}^{\prime}}(\xi)\right) d \lambda_{\xi}
$$

Из (3.24), (3.25) следует, что $I_{\nu, i} \leqslant \operatorname{const} I_{\nu}$, а из (3.23) - что $I_{\nu, i} \rightarrow 0$ при $\nu \rightarrow \infty$.

Далее, обозначим через $B(z, 1)$ шар с центром в $z$ радиуса 1 , а через $|B(1)|-$ его объем. Имеем

$$
\begin{aligned}
I_{\nu, i} \geqslant & \int_{B(z, 1)} \frac{\left|P_{\nu}(\xi) \varphi_{i}(\xi)-l(\xi) \varphi_{i}(\xi)\right|^{2}}{1+|\xi|^{2}} \exp \left(-2 H_{K_{i}^{\prime}}(\xi)\right) d \lambda_{\xi} \\
\geqslant & \exp \left(-2 \max _{B(z, 1)} H_{K_{i}^{\prime}}(\xi)\right) \cdot\left(\max _{B(z, 1)}\left(1+|\xi|^{2}\right)\right)^{-1} \\
& \times \int_{B(z, 1)}\left|P_{\nu}(\xi) \varphi_{i}(\xi)-l(\xi) \varphi_{i}(\xi)\right|^{2} d \lambda_{\xi} .
\end{aligned}
$$

В силу субгармоничности $\left|P_{\nu} \varphi_{i}-l \varphi_{i}\right|^{2}$ имеем

$$
\int_{B(z, 1)}\left|P_{\nu} \varphi_{i}-l \varphi\right|^{2} d \lambda \geqslant|B(1)|\left|P_{\nu}(z) \varphi_{i}(z)-l(z) \varphi_{i}(z)\right|^{2}
$$

в силу полуаддитивности $H_{K_{i}^{\prime}}$ имеем

$$
\max _{B(z, 1)} H_{K_{i}^{\prime}}(\xi)=\max _{|\zeta| \leqslant 1} H_{K_{i}^{\prime}}(z+\zeta) \leqslant H_{K_{i}^{\prime}}(z)+c,
$$

где $c=\max _{|\zeta| \leqslant 1} H_{K_{i}^{\prime}}(\zeta) ;$ наконец,

$$
\max _{B(z, 1)}\left(1+|\zeta|^{2}\right)=1+(|z|+1)^{2} \leqslant \text { const } \cdot\left(1+|z|^{2}\right) .
$$

Из вьшеизложенного следует

$$
I_{\nu, i} \geqslant \mathrm{const} \cdot \exp \left(-2\left(H_{K_{i}^{\prime}}(z)+\frac{1}{2} \ln \left(1+|z|^{2}\right)\right)\right)\left|P_{\nu}(z) \varphi_{i}(z)-l(z) \varphi_{i}(z)\right|^{2} .
$$

Поскольку $K_{i}^{\prime} \Subset \Omega_{i}$, сушествует промежуточньй компакт $K_{i}^{\prime \prime}, K_{i}^{\prime} \Subset K_{i}^{\prime \prime} \Subset \Omega_{i}$, такой, что

$$
H_{K_{i}^{\prime}}(z)+\frac{1}{2} \ln \left(1+|z|^{2}\right) \leqslant H_{K_{i}^{\prime \prime}}(z)+\text { const. }
$$

Таким образом,

$$
I_{\nu, i} \geqslant \text { const } \cdot \exp \left(-2 H_{K_{i}^{\prime \prime}}(z)\right)\left|P_{\nu}(z) \varphi_{i}(z)-l(z) \varphi_{i}(z)\right|^{2} .
$$

Это неравенство справедливо при любом z. Следовательно,

$$
\left\|P_{\nu} \varphi_{i}-l \varphi_{i}\right\|_{K_{i}^{\prime \prime}}^{2}=\sup \left|\frac{P_{\nu}(z) \varphi_{i}(z)-l(z) \varphi_{i}(z)}{\exp H_{K_{i}^{\prime \prime}}(z)}\right|^{2} \leqslant \text { const } \cdot I_{\nu, i} .
$$

Поскольку $I_{\nu, i} \rightarrow 0$ при $\nu \rightarrow \infty$, последовательность $P_{\nu} \varphi_{i}$ сходится к $l \varphi_{i}$ по норме $\|\cdot\|_{K_{i}^{\prime \prime}}$, а поскольку $K_{i}^{\prime \prime} \Subset \Omega_{i}$, сходимость будет иметь место в топологии $P\left(\Omega_{i}\right)$. Остается отметить, что вышеприведенное рассуждение справедливо для любого $i=1, \ldots, N$. 


\section{§4. Аппроксимационная теорема}

Пусть $\Omega_{1}, \ldots, \Omega_{q}$ - система выпуклых областей в $\mathbb{C}^{n}, \mathscr{H}=H\left(\Omega_{1}\right) \times \cdots \times$ $H\left(\Omega_{q}\right)$. В настояшем параграфе завершается доказательство аппроксимационной теоремы.

АППРОКСИМАЦИОННАЯ ТЕОРЕМА. Инвариантное подпространство $W$ peшений $f \in \mathscr{H}$ однородного уравнения векторной свертки $S * f=0$ допускает спектральный синтез.

1. Обильность главных подмодулей. Пусть $\Omega$ - система $\left\{\Omega_{1}, \ldots, \Omega_{q}\right\}$ выпуклых областей в $\mathbb{C}^{n}, \mathscr{P}=P\left(\Omega_{1}\right) \times \cdots \times P\left(\Omega_{q}\right)$.

Мы рассматриваем $\mathscr{P}$ как модуль над кольцом многочленов $\mathbb{C}[z]=\mathbb{C}\left[z_{1}, \ldots, z_{n}\right]$.

ТеОрема 4.1. Всякий главный подмодуль в ЯР является обильным.

ДокАЗАТЕЛЬСТво. Пусть $\varphi=\left(\varphi_{1}, \ldots, \varphi_{q}\right)$ - элемент в $\mathscr{P}, I$ - главный подмодуль, порожденный элементом $\varphi$. Подмодуль $I$ будет обильным (см. $\S 2$, п. 3 ), если всякий элемент в $\mathscr{P}$ вида $l \varphi$, где $l$ - целая функция, аппроксимируется в топологии $\mathscr{P}$ элементами вида $p \varphi$, где $p$ - многочлены. Включения $\varphi \in \mathscr{P}, l \varphi \in \mathscr{P}$ равносильны $2 q$ включениям

$$
\varphi_{1}, l \varphi_{1} \in P\left(\Omega_{1}\right), \quad \ldots, \quad \varphi_{q}, l \varphi_{q} \in P\left(\Omega_{q}\right)
$$

По теореме 3.1 существует последовательность многочленов $p_{n}$ такая, что $p_{n} \varphi_{i} \rightarrow l \varphi_{i}$ в топологии $P\left(\Omega_{i}\right), i=1, \ldots, q$. Это равносильно тому, что $p_{n} \varphi \rightarrow l \varphi$ в топологии $\mathscr{P}$. Теорема 4.1 доказана.

2. Завершение доказательства аппроксимационной теоремы. По предложению 2.2 аннуляторный подмодуль $I$ инвариантного подпространства $W \subset \mathscr{H}$ решений $f \in \mathscr{H}$ уравнения векторной свертки $S * f=0$ - главный. По теореме 4.1 этот подмодуль является обильным и, наконец, по теореме двойственности $(\S 1$, п. 6) подпространство $W$ допускает спектральный синтез. Аппроксимационная теорема доказана.

\section{Список литературы}

1. Schwartz L. Théorie générale des fonctions moyenne-périodique // Ann. of Math. (2). 1947. V. 48. № 4. P. 857-929.

2. Ehrenpreis L. Mean periodic functions // Amer. J. Math. 1955. V. 77. № 2. P. 293-328.

3. Malgrange B. Existence et approximation des solutions des équations aux dérivées partielles et des équations de convolution // Ann. Inst. Fourier (Grenoble). 1955-1956. V. 6. P. 271-354.

4. Красичков- Терновский И. Ф. Однородныте уравнения типа свертки на выпуклых областях // Докл. АН СССР. 1971. Т. 197. С. 29-31.

5. Напалков В. В. Уравнения свертки в многомерных пространствах. М.: Наука, 1982.

6. Юлмухаметов Р. С. Однородные уравнения свертки // Докл. АН СССР. 1991. Т. 316. №2. C. $312-315$.

7. Кривошеев А. С., Напалков В. В. Комплексньй анализ и операторы свертки // УМН. 1992. T. 47. №6 (288). C. 3-58.

8. Юлмухаметов Р. С. Асимптотика плюрисубгармонических функций // Препринт. Уфа, 1986.

9. Юлмухаметов Р. С. Однородные уравнения свертки // Препринт. Уф̆а, 1990. 
10. Sibony $N$. Approximation polynomiale ponderee dans un domaine d'holomophy de $\mathbb{C}^{n} / /$ Ann. Inst. Fourier (Grenoble). 1976. V. 26. № 2. P. 71-99.

11. Себаитьлн-и-Силва ЖК. О некоторых классах локально-выпуклых пространств, важных в приложениях // Математика. Сб. пер. 1957. Т. 1. №1. С. 60-77.

12. Эдвардс Р. Функциональный анализ. Теория и приложения. М.: Мир, 1969.

13. Красичков-Терновский И. Ф. Фундаментальный принцип для инвариантных подпространств аналитических функций. II // Матем. сб. 1997. Т. 188. №6. С. 57-98.

14. Красичков-Терновский И. Ф. Спектральный синтез и локальное описание для многих переменных // Изв. РАН. Сер. матем. 1999. Т. 63. № 4. С. 101-130.

15. Шишкин А.Б. Спектральньй синтез для систем дифференциальных операторов с постояннњми коэффициентами. Теория двойственности // Матем. сб. 1998. Т. 189. №9. C. $143-160$.

16. Красичков-Терновский И. Ф. Спектральньй синтез аналитических функций на системах выпуклых областей // Матем. сб. 1980. Т. 111. № 1. С. 3-41.

Институт математики с ВЦ РАН, Уфа

Поступила в редакцию

E-mail: kra@imat.rb.ru

25.06.2003 